\title{
The endurance of flash-frozen biologics can be improved allowing high-resolution electron microscopy tomography on individual proteins
}

Andrea Fera ( $\sim$ andrea.fera@gaaconsultinggroup.com )

GAA Inc.

\section{Biological Sciences - Article}

Keywords: Isolated Protein Constructs, Flash-frozen Rigid Biopolymers, Atomic Coordinates, Proteinprotein Interactions, Cryo-electron Microscopy Samples

Posted Date: June 2nd, 2021

DOI: https://doi.org/10.21203/rs.3.rs-567961/v1

License: (9) This work is licensed under a Creative Commons Attribution 4.0 International License.

Read Full License 


\title{
The endurance of flash-frozen biologics can be improved allowing high-resolution electron microscopy tomography on individual proteins
}

\author{
Andrea Fera, Principal Scientist \\ Geri Anderson \& Associates, Inc., 12532 Westland Ct, Fulton, MD 20759 \\ andrea.fera@gaaconsultinggroup.com
}

Here surprising results are shown demonstrating a workflow possibly able to exploit the discreet nature of interactions between high-energy electron beams and matter. Isolated protein constructs have been imaged after an original temperature-curing in vacuum, introduced recently for flash-frozen rigid biopolymers, and here applied to flash-frozen oligomers of viral origin. These results, if confirmed, may extend to plastics and bio-oligomers the access to atomic coordinates in one experimental session, when imaged by electron microscopy. Which is the case when imaging semiconductors or other solid materials, provided that the samples are not damaged by the interaction with accelerated electron beams in vacuum. Therefore, potentially, this workflow introduces the possibility of achieving atomic resolution in only one experiment with data only about one individual protein, maybe out of thermodynamic equilibrium. Such data are vital to understand protein-protein interactions. Finally, this workflow offers the possibility, new to cryo-electron microscopy samples, to store a sample indefinitely under liquid nitrogen for imaging the same molecules in more than one experimental session.

Introduction The restricted stability of frozen biological samples to accelerated electron beams in vacuum has been the major limiting factor for high-resolution studies by electron microscopy (EM), since the introduction of fast-freezing immobilization techniques. While flashfreezing allows to immobilize proteins in their instantaneous configuration, when imaging with accelerated electron beams samples degrade rapidly. Such situation has limited accurate determinations because EM signal follows Poissonian statistics, where the error is low enough when the number of events (i.e. electrons scattered) is large enough (i.e. electron dose). Lately, averaging the data from many thousands experiments has been introduced for bypassing this problem, but the low signal-to-noise $(\mathrm{S} / \mathrm{N})$ of individual images used for calculating the average 
structures limits applications. Until today no viable solutions to increase the stability of such frozen samples under electron-beams has been proposed.

Previously introduced only for rigid cellular biopolymers, here is an application to EM imaging of viral proteins of a phenomenon pertaining the interaction of high-energy electron beams with matter [1]. Results here will focus on electron microscopy of isolated, assembled aggregates of the R18L mutant of the CA matrix protein of the Human Immunodeficiency Virus (HIV-1). If confirmed, this behavior could allow to use a new workflow for acquiring, in one single experimental session, structural data at sub-nanometer resolution pertaining flash-frozen viral proteins. Therefore atomic-scale structures of individual (not averaged) proteins or protein aggregates, including molecules out-of-equilibrium, would become available. Such advances may help the introduction of new drugs aiming at interfering with targeted mechanisms, like viral entry.

The data presented here can be explained in the framework of the quantization of the energy of the atomic (electronic) states. Because of this quantization, it is widely accepted [2] that the probability of interference of atomic electrons with accelerated incoming electrons $\left(\mathrm{P}_{\mathrm{el}}\right)$ is inversely proportional to the difference between the kinetic energy of the incoming electrons $\left(\mathrm{E}_{\mathrm{keV}}\right)$ and the ionization energy $\left(\mathrm{E}_{0}\right)$ of the atomic electrons before interaction, or:

$$
\left.\mathrm{P}_{\mathrm{el}} \propto\left(\mathrm{E}_{\mathrm{keV}}-\mathrm{E}_{0}\right)^{-1} \quad \text { (eq. } 1\right)
$$

This dependence is the underlying motivation of the very high elemental sensitivity of modern chemical surface analysis techniques, like Auger spectroscopy. From eq. 1, when the term between brackets grows, $\mathrm{P}_{\mathrm{el}}$ decays monotonically.

Using this framework, the results are shown from an original workflow able to output flashfrozen proteins resistant to sustained electron irradiation. The physical origin of this behavior remains a matter of debate: here experimental findings are made known to the wide scientific community in order to accelerate its fruition and, possibly, progress. A possible interpretation is attempted.

Recently it has been demonstrated that a flash-frozen sample of rigid biopolymers [1] (previously stained by exposing it to a transition metal salt), when inserted in high-vacuum conditions for a long enough time, while maintained at controlled temperatures (always wellbelow freezing point), are able to sustain, unaltered, over 1.5 hours of illumination from a120 $\mathrm{keV}$ electron beam. In fact, if comfirmed, such data may shed light on the interactions of the 
individual subunits in solution, and on the mechanisms of formation of such biopolymers, invitro. For the present work, that result sets the stage to test such stability on viral proteins (and oligomers) to sustained irradiation from high energy electron beams.

Here we focus on a similar high-vacuum curing of assembled, flash-frozen proteins extracted from HIV-1, and maintained at all times at temperatures well below freezing inside a transmission electron microscope (TEM), before illuminating with an accelerated electron beam. Assembled proteins cured in this way are demonstrated to become resistant to the rigors implicit to obtaining the high-S/N images necessary to reconstruct a high-resolution tomogram (i.e. able to show signal variations of the order of two voxels size). It is further shown that this behavior is independent of pre-staining the sample with heavy-metal cross-linkers. In these conditions, i.e. in the high-vacuum atmosphere inside a TEM, and virtually in absence of water molecules in the beam path, it has also been possible to image un-stained biological samples (albeit with a very sensitive camera) using a beam focused within $\sim 300$ nanometers from zero defocus level. These results seem to indicate the possibility to use this method for studying directly (exploiting amplitude contrast) protein-protein interactions, like e.g. viral spikes interacting with cell receptors.

Results Figure 1 shows two images of the same grid with deposited assembled mutant R18L of the CA protein of the HIV-1 capsid, kindly donated by Owen Pornillos and Barbie GanserPornillos of the Medical School at the University of Virginia (UVA) in Charlottesville, VA. All images shown here have been obtained with a non-aberration-corrected TEM, operating at 200 $\mathrm{keV}$ at the modern TEM facility of the Medical School. The data shown in figures 1 and 2 have been obtained using a magnification leading directly a pixel size of $0.1745 \mathrm{~nm}$. The two images in Figure 1 have been obtained, respectively, at the beginning of the tomography series (left), and after 121 images at varying angles (right). The images of stained samples reported here have been obtained manually focusing at increasing tilt angles (Materials\&Methods). 


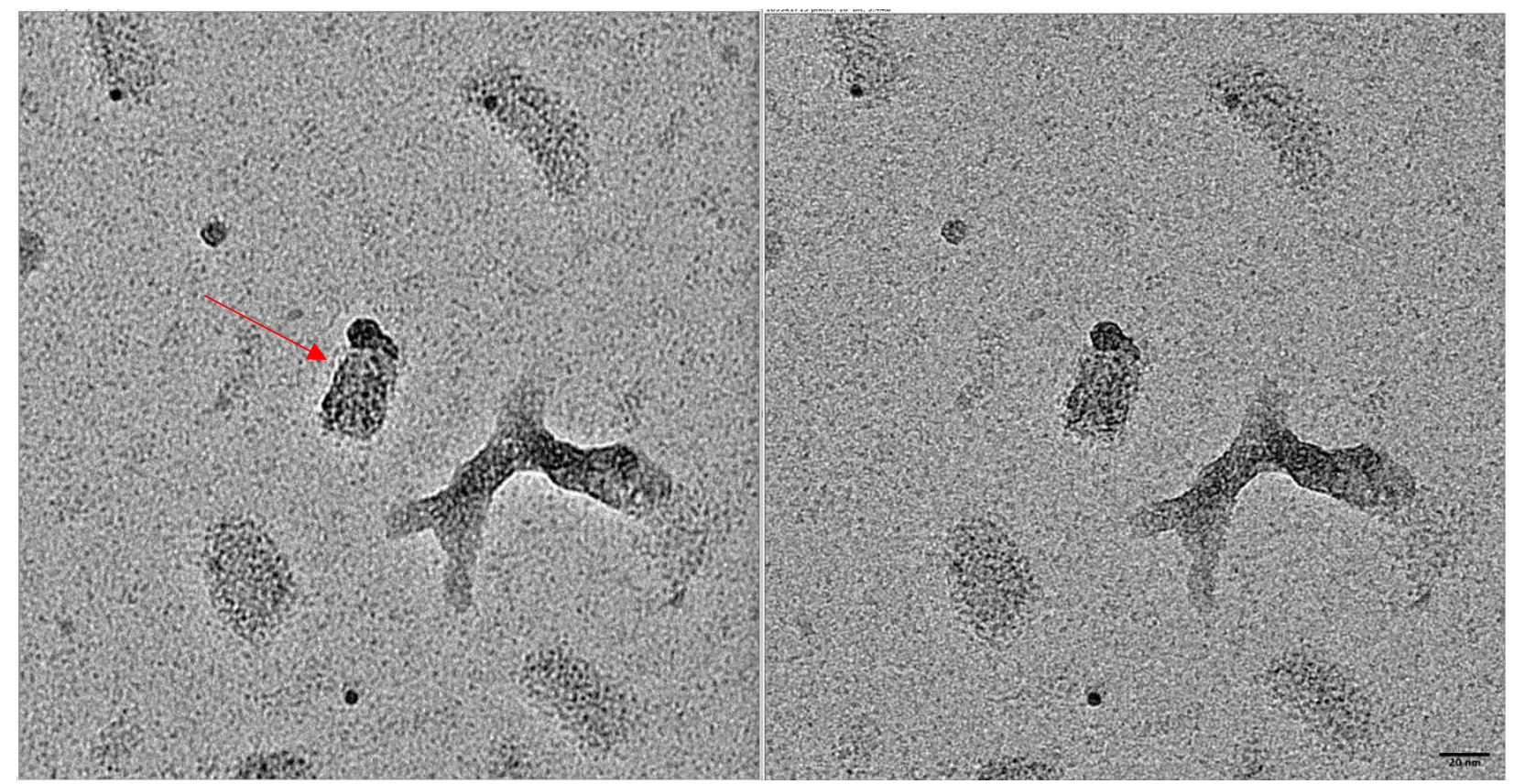

Figure 1: Successive images of the same assembled R18L-CA protein at different stages of the tomography acquisition. (Left) at the beginning; (right) after a full acquisition for a tomogram with 121 images.

From a closer analysis of the images, apart for differences in defocus, it is apparent that the two images show the same details. The area shown is a small part of the 4096x4096 field of view, in order to appreciate the signal invariance at high magnification. Note the $6 \mathrm{~nm}$ colloidal gold beads used as fiduciary markers. Since each image had to be focused manually, small differences of defocus were uncontrollable at the time of this experiment. More trials with automatic software are on the way. Our current interpretation is that this result is a consequence of the curing of the sample prior to illumination with the electron beam.

Figure 2 shows (with inverted contrast) three perpendicular virtual sections (from left to right, respectively, the $\mathrm{XY}$, the $\mathrm{XZ}$ and the $\mathrm{YZ}$ ) calculated from the corresponding tomogram of the object indicated by a red arrow in figure 1.

Figure 3 shows analogously XZ, XY and YZ virtual sections extracted from another tomogram measured from an unstained assembled aggregate of the same R18L mutant of CA-HIV-1 [3,4], imaged and shown with magnification leading directly to a pixel size of $0.1112 \mathrm{~nm}$. The pictures demonstrate that as well this sample, subjected to the same temperature curing preceding illumination, is not collapsed (figure 3 is shown with inverted contrast, and binned 4X). For comparison, a tomogram of the same constructs of R18L-CA-HIV-1 negative stained and air-dried on grids, shows collapsed structures of $\sim 7 \mathrm{~nm}$ height (data not shown), as found before for negative stained enveloped virus [5]. Figures 2 and 3 show structures clearly not collapsed 
while corresponding renderings (figures 4 and 5), realized fixing a unique threshold for the isosurface, reveals the typical hexagonal motif of this protein. Held together by dipole-dipole interactions, the hexagonal motifs in these sample are conserved especially in the shape of the central cavities of the constituting 'donuts'.
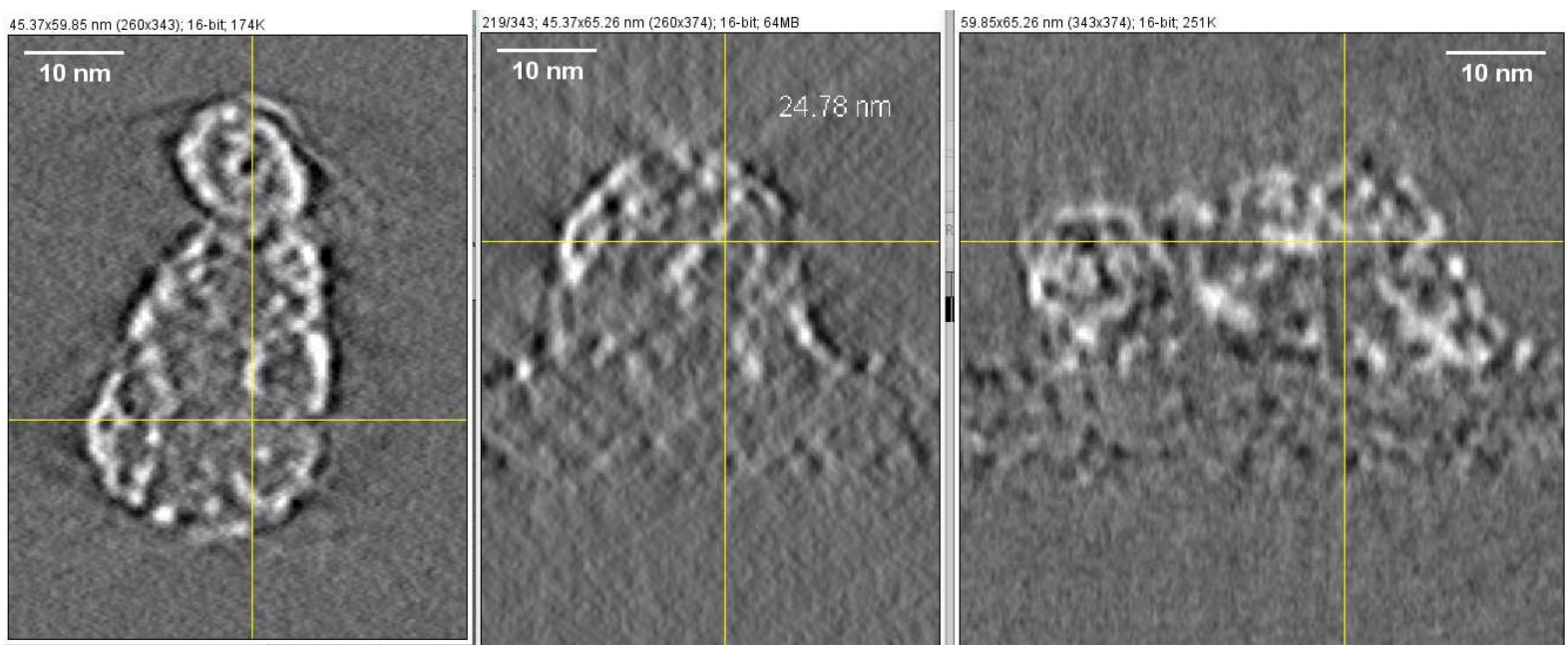

Figure 2: Perpendicular virtual sections extracted from a tomogram calculated from imaging as described an individual positive stained assembled construct of the R18L-CA-HIV-1 protein. Left: XY plane; center : XZ plane and right: YZ plane. The yellow cross indicate the same point in the three planes for clarity. Contrast inverted. Image unbinned, voxel size: $0.1745 \mathrm{~nm}$.


Figure 4: Perpendicular Virtual sections ( $X Z, X Y$ and $Y Z$ planes respectively) extracted from a tomogram measured imaging an unstained individual aggregate of the R18L mutant of the CA-HIV-1 protein. Images acquired at pixel size $1.112 \AA$ but shown after binning by 4 using the Scale function (value 0.25) of Fiji-ImageJ. The yellow marker indicates the same point in the three projections. A fiduciary marker is seen in the XY projection. Contrast inverted. Scale bars: $10 \mathrm{~nm}$. 
The common factor in all experiments seems to be the fact that free water molecules are removed by sublimation from the sample during curing inside the TEM before illuminating with accelerated electrons. This is accomplished always well below freezing point, but at temperatures significantly higher than the liquid nitrogen (Materials \& Methods) [6,7]. The absence of ice was indicated by the absence of opaque areas that progressively disappear when illuminated by the electron beam. When ice was still present, those areas were not chosen for further elaboration. In order to complete the present illustration of results, Figures 4 and 5 show corresponding 3D renderings from the tomograms acquired with the samples shown above. These renderings have been obtained with the freeware software TOMVIZ (Cornell and Michigan University), using the 'contour' tool that renders isosurfaces.
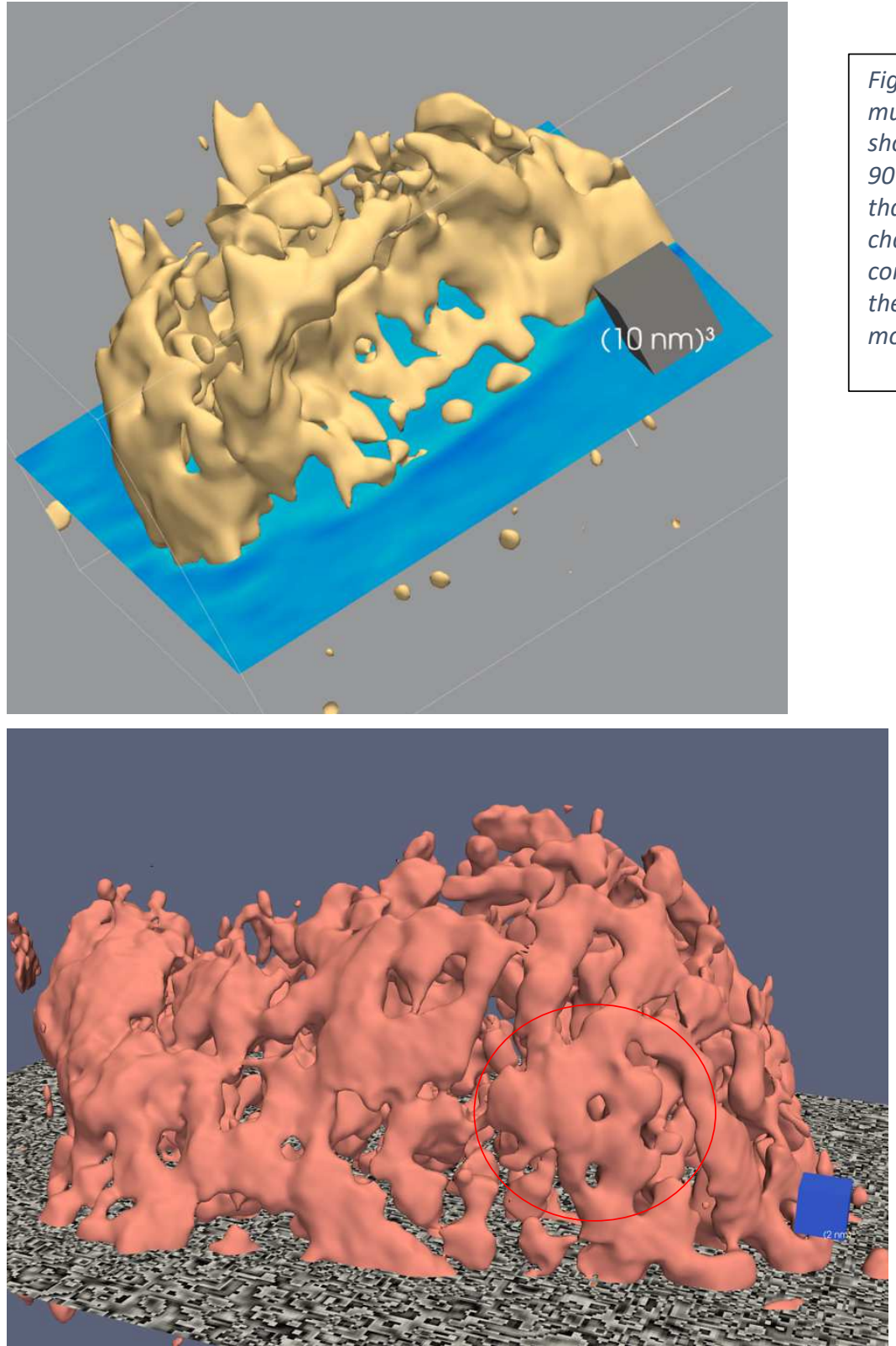

A Fera
Figure 5: $3 D$ rendering of the aggregated $R 18 L$ mutant of CA-HIV-1 protein, imaged unstained and shown in figure 3; this sample has been imaged at$90^{\circ} \mathrm{C}$. The binning $4 X$ results in a smoother surface than the corresponding stained sample. Note the characteristic size and shape of the holes of the constituting units of this aggregate determined by the strong dipole-dipole interactions between monomers.
Figure 6: $3 D$ rendering of the $R 18 L$ mutant of the assembled CA-HIV-1 shown in figure 1 and 2. This sample has been measured after staining with UA followed by a quick washing step before flash-freezing; tomogram acquired at $-92^{\circ} \mathrm{C}$. The known shape of the hexagonal motives is recognizable (red circle). The blue cube is meant as size reference: size $=2 \mathrm{~nm}$; it has been placed roughly in the same plane as the objects inside the red circle. 
Discussion

At present, the origin of these surprising results is not clear, and artefacts may not be completely ruled out at this stage. The main, current interpretation is that this result benefits from the instability of free water molecules in vacuum, i.e. their tendency to readily sublimate. Water molecules not bound to chemically polar groups (of a proteins or of the stain) are well-known to sublimate at the temperatures used during curing [6,7] (and just a little slower at liquid nitrogen temperatures) if maintained in a high-vacuum environment, like the one intrinsic to a TEM column. In the conditions chosen, proteins stay solid while water sublimation is faster (reaching a rate of a few $\mathrm{nm} /$ minute) [6].

At the current level of understanding, such considerations may contribute as well to tentatively explain some behaviors commonly observed when imaging (unstained) flash-frozen but hydrated biological samples. In fact, unbound water molecules likely increase the rate of absorption of the parasitic radiation generated by the electron beam while passing through a biological sample. Indeed, considering the energy variation experienced by electrons deflected while penetrating a cryo-biological sample, the biggest majority of events are [2] 'small' energy exchanges, likely of the order of $1 \mathrm{eV}$ or a fraction. Actually, $1 \mathrm{eV}$ (corresponding to $6000 \mathrm{~cm}^{-1}$ ) is actually about double the center of the strongest (not the unique) Raman absorption peak for water molecules [8]. Water indeed absorbs strongly at Raman frequencies, which results in atomic vibrations. This strong Raman adsorption of water is not shared by $\mathrm{R}-\mathrm{OH}$ groups, because of their different symmetry. In fact, this tentative theory may suggest a reason why ice degrades (melts) rather quickly after illumination with an electron beam; following this reasoning, isolated proteins would remain untouched by the electron beam, as in figure 1 .

For the sake of argument, if adopting this reasoning, the higher bound (i.e. the temperature limit where this attempted mechanism loses validity) would be where the proteins are not rigid anymore, i.e. at the melting temperature of proteins. In other words, temperature has to be kept at all times well below the melting point of proteins, in order to guarantee the absence of obvious distortions. For all the experiments shown here, temperature has been kept at all times below or around (minus) $-90^{\circ} \mathrm{C}$. Which value has been chosen studying the phase diagram of ice and the known data pertaining the speed of sublimation of ice, in vacuum, well below $0^{\circ} \mathrm{C}$, a situation incurred at times in studies of interstellar space or planets without an atmosphere $[6,7]$. It is well-known that the interaction of accelerated electrons with an atom is not a continuous spectrum at all energies of the incoming electrons. It is instead a discrete sequence of absorption 
lines (see: Auger Spectroscopy) depending on the energy of the incoming electrons, that has to match the ionization potential of one atom in the material invested by the radiation. In other words, $\mathrm{P}_{\mathrm{el}}$ has to always be taken into account [2] since the atomic electron energy levels are quantized. Therefore, examining the energy levels of the inner core electrons of the heaviest element in these samples, Uranium has the 1s core energy level in the range of $\sim 105.600 \mathrm{keV}$ [9]. Only electrons with energies within a few percent will be able to interact with the $1 \mathrm{~s}$ electrons of Uranium, where the corresponding level in Uranyl cannot be at much higher energies since, when using $120 \mathrm{keV}$ electrons, no interaction has been observed [1]. All other elements in these samples have electron levels of less energy, so further from the energy of the electrons in the beam. Other possible candidates are the higher energy levels of the nuclei, but these are at least at 3 orders of magnitude higher energy. In these conditions, as found experimentally, elementary physics models [2] seem to predict that electrons would pass through these sample and only elastic type of scattering interactions would happen. As it seems indicated by the possibility of measuring figure 1 indeed.

In summary, this invariance seems to be due to the fact that no energy levels in the sample are near $200 \mathrm{keV}$, i.e. the energy of the accelerated electrons from the electron microscope. One other remarkable fact stemming out of these trial measurements is that unstained, unaveraged protein molecules can be observed in vacuum without high defocus, at least when virtually in absence of free water. The details shown (figure 4) are of the order of $0.5 \mathrm{~nm}$, as evinced from the central cavities of the hexagonal units at the center of every 'donut' in the renderings of figure 4 , whose datafile is binned $4 \mathrm{X}$. Without binning, the rendering of Figure 5 shows a structure with higher level of details but, remarkably, very similar central cavities of the motifs. As expected since these interactions are strong dipole-dipole interactions stronger than thermal energy at room temperatures. Further elaborations of similar samples, and possibly some averaging, may allow to better reproduce an underlying symmetry, as found by cryo-EM $[3,4]$.

\section{Materials and Methods}

R18L mutants of the CA protein of HIV-1 have been kindly donated by Barbie Ganser-Pornillos and Owen Pornillos. They have been assembled in the buffer provided immediately before deposition on a cupper formvar or formvarcarbon grid, 200 or 300 mesh (EMS Sciences). The deposition has been realized suspending the 
grid (with the shiny side facing up) on a self-closing tweezer (EMS-Sciences). Using a standard pipette, a $\sim 6 \mu$ drop containing the fresh assembled R18L CA protein has been deposited on a grid, on the bench, waiting about 1.5 minutes for the second step. Then, after wicking the excess solution, a $\sim 6 \mu \mathrm{l}$ drop of $2 \%(\mathrm{w} / \mathrm{v})$ Uranyl Acetate is deposited on the grid for about 45 seconds. After wicking the excess solution, another drop of solution containing $6 \mathrm{~nm}$ colloidal gold spheres is deposited on the sample for $\sim$ a minute, followed by mounting the sample vertical in the 'guillotine' available in Dr. Dryden's lab for quickly plunge-freezing samples by plunging in liquid ethane, after blotting carefully the excess solution from the vertical grid.

Analogously, for the unstained sample, a $\sim 6 \mu$ drop containing R18L assembled in exactly the same way has been deposited on a Formvar-C 200 mesh grid, for about 2 minutes, wicking the solution excess, immediately before adding $6 \mu \mathrm{l}$ of the same Asurion solution containing $6 \mathrm{~nm}$ colloidal gold. After about a minute, the grid with R18L has been mounted vertical in the aforementioned guillotine, wicking the solution in excess while vertical, and immediately plunge-freezing in liquid ethane.

Once the samples were flash-frozen, they have been inserted in a Gatan Cryo holder and mounted inside the TF20 Tecnai, making sure that they were in contact with liquid nitrogen all the time. Once connected to the sample holder inserted in the TEM, the Gatan controller measured typically $-172^{\circ} \mathrm{C}$. Then a temperature ramping cycle has been started setting as final temperature $-100^{\circ} \mathrm{C}$ at a speed of $3^{\circ} \mathrm{C} / \mathrm{min}$. Once set temperature was reached, or immediately before to prevent overshooting, the speed of heating has been set to $1^{\circ} \mathrm{C} / \mathrm{min}$ and the final temperature has been set to $-90^{\circ} \mathrm{C}$ or $-92{ }^{\circ} \mathrm{C}[6]$. Temperature has been maintained constant during EM imaging afterwards at varying angles, making sure to record as well a second image at $0^{\circ}$ tilt at the end of the experiment, guaranteeing that the sample has not been altered by the sustained irradiation. Occasionally the temperature has been raised, before acquisition, to as high as $-28^{\circ} \mathrm{C}$ in order to speed up the sublimation of the solvent ; apart for a more complete sublimation of the solvent, a similar behavior (the proteins seemed solid during the acquisition at the same temperature) has been observed. Nevertheless, data at temperatures higher than $-90^{\circ} \mathrm{C}$ are still under evaluation and have not been used here.

Tomograms have been acquired in two ways: (1) varying the angle by hand $\pm 60^{\circ}$ acquiring high S/N images (like figure 1 ) every $1^{\mathrm{o}}$; (2) using the SerialEM software to vary the angle in the same range. This allowed to standardize some settings like average defocus, which was set at - 
$300 \mathrm{~nm}$ or to zero in the unstained experiment reported. Each image further used for calculating a high-resolution, high $\mathrm{S} / \mathrm{N}$ tomogram (necessary to examine details of the order of the voxel size) has been acquired with at least $8000 \mathrm{e}^{-} / \mathrm{nm}^{2}$. Therefore, including also the images for centering, a complete tomography acquisition is realized with an electron dose of the order of $1.4 \cdot 10^{6} \mathrm{e}^{-} / \mathrm{nm}^{2}$. After imaging, samples have routinely been stored under liquid nitrogen. When necessary, the same area has been re-imaged the next day, sometimes at liquid nitrogen temperature if no significant ice was deposited on the sample during handling outside the electron microscope. All samples imaged are currently still stored under liquid nitrogen in the facility of the Medical School of UVA in Charlottesville, VA, where all experiments have been carried out.

Competing Interests: The experimental procedures here described have been all incorporated in U.S. Provisional Patent Application No. 63/074,052 filed on September 3, 2020.

\section{Acknowledgements:}

All the experiments here reported have been carried out using the microscopes and equipment/personnel of the Medical School in the University of Virginia Charlottesville, assisted by Dr. K. Dryden.

The prompt response of the Ganser-Pornillos group (especially Barbie and Owen) has allowed this work to be completed in a rather short time.

AF acknowledges the help of Mira Shapiro refining the grammar of this manuscript.

For discussions at various stages of the preparation of this manuscripts AF acknowledges Dr. P. Ercius, of the Molecular Foundry at the Lawrence Berkeley National Laboratory in Berkeley, California. 
REFERENCES:

[1] Fera A. et al., Microsc. Microanal. 23 (Suppl 1), Pages. 1114-5, 2017

[2] S. Tavernier, Experimental Techniques in Nuclear and Particle Physics, Springer 2010.

[3] Ganser-Pornillos, von Schwedler et al (2004) J Virology 78 : 2454-2464; Ganser-Pornillos et al. (2007) Cell 131: $70-79$

[4] Pornillos O, Ganser-Pornillos BK, Yeager M. Atomic-level modelling of the HIV capsid. Nature. 2011 Jan 20;469(7330):424-7. doi: 10.1038/nature09640. PubMed PMID: 21248851; PubMed Central PMCID: PMC3075868.

[5] A. Fera, J.E. Farrington, J. Zimmerberg, T.S. Reese, Microsc Microanal, 18 (2012) 331-335.

[6] H. P. Zingsheim, Journal of Microscopy, Vol. 133, Pt 3, March 1984, pp. 307-312. Sublimation rates of ice in a cryo-ultramicrotome

[7] W. Wagner, T. Riethmann, R. Feistel and A.H. Harvey, New Equations for the Sublimation Pressure and Melting Pressure of H2O Ice Ih, J. Phys. Chem. Ref. Data, Vol. 40, No. 4, 2011; G. Jancso, J. Pupezin and W. A. Van Hook, The Vapor Pressure of Ice between $+10^{-2}$ and $10^{+20}$, The Journal of Physical Chemistry, Vol. 74, Vo. 15, 1970. D. E. J. Hobley, J. M. Moore and A. D. Howard, How rough is the surface of Europa at lander scale? , 44th Lunar and Planetary Science Conference (2013) p.2432

[8] M. Pastorczak*, M. Kozanecki, and J. Ulanski, Raman Resonance Effect in Liquid Water, J. Phys. Chem. A 2008, 112, 43, 10705-10707 https://doi.org/10.1021/jp805369p

[9] E. Clementi and D.L.Raimondi, J. Chem. Phys. 1963, 38, 2686.; E. Clementi, D.L.Raimondi, and W.P. Reinhardt, J. Chem. Phys. 1967, 47, 1300. https://www.webelements.com/uranium/atoms.html, see: Uranium 1s binding energy. 